\title{
ANÁLISE EXPERIMENTAL E NUMÉRICA DO COEFICIENTE DE RESTITUIÇÃO EM SIMULAÇÕES CFD DOS REGIMES DE ESCOAMENTO EM UM MOINHO DE BOLAS
}

\author{
${ }^{1}$ VITOR STRAATMANN, ${ }^{2}$ MARCELA V. C. MACHADO, ${ }^{3}$ MARCOS A. S. BARROZO, E \\ ${ }^{4}$ CLAUDIO R. DUARTE
}

${ }^{1}$ Bolsista de iniciação Científica PIBIC/CNPq/UFU, do curso de Engenharia Química

${ }^{2}$ Doutoranda do Programa de Pós-Graduação em Engenharia Química da UFU.

${ }^{3,4}$ Professor da Faculdade de Engenharia Química da UFU/MG

E-mail para contato: claudiofequfu@gmail.com

\begin{abstract}
RESUMO - Em um moinho de bolas, o bom entendimento do fenômeno de colisão entre corpos moedores é importante para obter-se boa eficiência na operação de moagem. A ocorrência de determinado regime de movimentação dentro do moinho depende do arranjo de variáveis operacionais, entre outros. Deste modo, este estudo propõe uma análise da influência da parcela da energia envolvida em uma colisão que é restituída na forma de energia cinética, sobre o movimento dos corpos moedores, sendo esta definida pelo coeficiente de restituição. Em uma metodologia experimental, foi determinado o valor do coeficiente de restituição entre duas esferas pela análise da colisão entre as mesmas utilizando-se uma câmera de alta velocidade. Além disso, utilizou-se de simulação em CFD através do software FLUENT $^{\circledR}$ e do Modelo Euleriano Granular Multifásico para simular um moinho de bolas com suspensores. Os valores de coeficiente de restituição simulados foram de 0,50, 0,60 e 0,70 foram avaliados nas velocidades correspondentes ao regime de cascata $(17,0 \mathrm{rpm})$, catarata $(31,7 \mathrm{rpm})$ e centrifugação $(74,6 \mathrm{rpm})$, observadas previamente. Os resultados indicaram pouca influência do coeficiente de restituição no movimento de carga moedora em simulações computacionais, exceto para elevadas velocidades de rotação empregadas, que resulta no regime de centrifugação. Além disso, o valor experimental obtido na determinação do coeficiente foi satisfatoriamente compatível com os resultados obtidos em simulação.
\end{abstract}

\section{INTRODUÇÃO}

O movimento da carga moedora em um moinho de bolas afeta significativamente a eficiência da operação de moagem através da ocorrência dos fenômenos de quebra. A depender de condições operacionais como, a velocidade de rotação, o nível de enchimento do moinho e características da geometria do equipamento, certos regimes de escoamento irão surgir no interior do tambor rotatório.

Os principais regimes de movimentação observados no interior de um tambor rotatório podem ser classificados como rolamento, cascata, catarata e centrifugação (Figura 1). Os regimes de cascata e catarata são os mais empregados na operação de moinhos de bolas, pois 
vão favorecer a quebra das partículas (KAPUR et al., 1992), enquanto que o regime de centrifugação deve ser evitado na operação de moagem.

Figura 1: Regimes de escoamento transversal em tambor rotatório: a) rolamento; b) cascata; c) catarata; d) centrifugação.

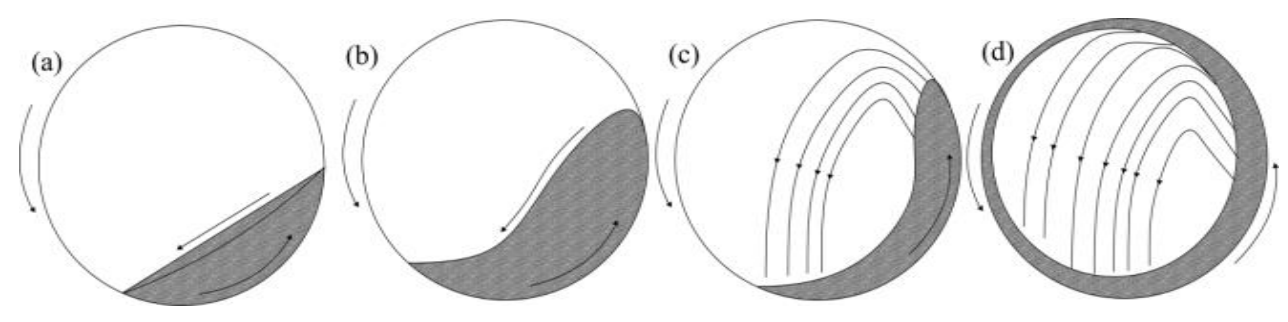

O estudo do comportamento dos corpos moedores em um moinho de bolas pode ser realizado por abordagens numéricas e/ou experimentais. Em estudos numéricos, a Fluidodinâmica Computacional (CFD) é uma ferramenta amplamente utilizada, pois permite obter informações em qualquer ponto do processo, o que nem sempre pode ser obtido em abordagens experimentais. Os métodos numéricos podem ser aplicados pela aproximação Lagrange ou pela aproximação Euler-Euler.

\subsection{Abordagem Euleriana}

$\mathrm{Na}$ abordagem Euler-Euler, a fase dispersa é considerada como um fluido, logo o sistema é interpretado como duas (ou mais) fases contínuas e interpenetrantes. Dessa forma, a fase granulada, juntamente com a fase fluida, é modelada em um campo Euleriano. Muitos são os estudos sobre a dinâmica de partículas sólidas no interior de tambores rotatórios (NASCIMENTO, 2015; SANTOS, 2013;). Como exemplo de aplicação, tem-se o entendimento da dinâmica dos corpos moedores em um moinho de bolas e seus regimes de escoamento.

Quando o sistema é composto por partículas e fluido, como é o caso deste trabalho, as propriedades físicas da fase particulada são interpretadas utilizando a Teoria Cinética Granular, e pelo Modelo Euleriano Granular Multifásico (MACHADO, 2016).

A Teoria Cinética Granular utiliza de equações empíricas para modelar as interações entre partículas e, desta forma, descrever o comportamento do material. No estudo das colisões entre partículas surge, então, a necessidade de um parâmetro que considere a dissipação de energia associada a colisão, sendo este o coeficiente de restituição.

Os valores possíveis para o coeficiente de restituição variam entre 1 para colisões perfeitamente elásticas, ou seja, não há dissipação de energia e 0 para colisões perfeitamente inelásticas, nas quais toda a energia cinética associada ao choque é dissipada.

O software FLUENT® apresenta este parâmetro como default (padrão) igual a 0,9 e em estudos de simulação DEM ainda foram utilizados valores de 0,6 e 0,66 para esferas de açocromo por Rajamani e Venugopal (2001) e Hlungwani et al. (2003) respectivamente. Normalmente, a medida experimental do coeficiente de restituição não é realizada e, em estudos de simulação, valores deste coeficiente encontrados na literatura são adotados ou mesmo arbitrariamente assumidos.

Sendo assim, este trabalho tem como objetivo a determinação experimental do coeficiente de restituição entre duas esferas de moagem e verificar se este valor é suficiente 
para descrever em simulação o regime de escoamento condizente com o regime encontrado na literatura paras as velocidades determinadas. Além disso, foi realizada uma investigação sobre a influência do coeficiente de restituição sobre o movimento dos corpos moedores em um estudo de simulação CFD.

\section{MATERIAIS E MÉTODOS}

\subsection{Abordagem Experimental}

Para o estudo experimental, utilizou-se de uma câmara de alta velocidade com capacidade de filmar até 2000 quadros por segundo, a qual foi disposta frontalmente ao local de colisão, tal como ilustra Figura 2.

Figura 2: Visão esquemática do método experimental utilizado para medida do coeficiente de

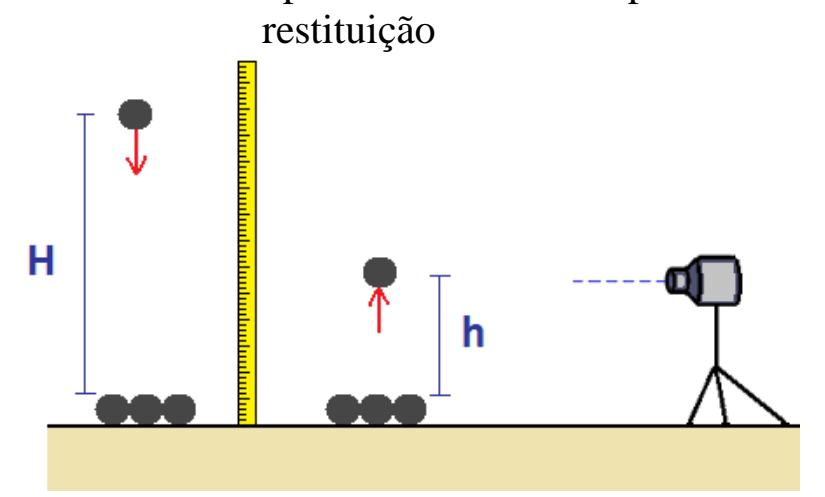

Esferas de aço-cromo, de densidade $\rho_{\mathrm{s}}=7890 \mathrm{~kg} / \mathrm{m}^{3}$ e diâmetro $25 \mathrm{~mm}$, foram utilizadas para determinação do coeficiente de restituição. A esfera era solta a uma altura(H) e a altura máxima que esta atingia após o ressalto (h) foram observadas através das gravações feitas pela câmera de alta velocidade utilizada.

Os valores do coeficiente de restituição $\left(e_{s s}\right)$ foram obtidos pela Equação 1:

$$
e_{S S}=\sqrt{\frac{h}{H}}
$$

A altura inicial que a esfera foi abandonada variou entre 15 e $30 \mathrm{~cm}$. Os valores de altura máxima atingida após o ressalto só foram consideradas nas medidas em que a esfera aparece ressaltando verticalmente, com mínimo ou nenhum desvio para a horizontal.

\subsection{Abordagem numérica}

O primeiro passo para a realização de uma simulação em CFD é a criação de malha computacional através do software GAMBIT ${ }^{\circledR}$. A malha é do tipo regular tridimensional, composta por 130000 células e possui as dimensões de um moinho de bolas com 12 suspensores quadrados, diâmetro de $30 \mathrm{~cm}$ e comprimento de $8,1 \mathrm{~cm}$. Esferas de aço-cromo com as mesmas características das esferas utilizadas na determinação experimental do coeficiente de restituição foram utilizadas para simular os corpos de moagem no interior do moinho de bolas. 
As simulações foram realizadas utilizando o software FLUENT ${ }^{\circledR} 14.0$ e considerou-se o padrão de mistura descrito pelo Modelo Euleriano Granular Multifásico. Para investigação das interações partícula-partícula e partícula-parede foi aplicada a Teoria Cinética Granular.

Foram adotados três valores de coeficiente de restituição para serem analisados na simulação numérica: 0,50, 0,60 e 0,70. Estes valores correspondem a uma faixa dos resultados obtidos experimentalmente, conforme será mostrado posteriormente. As simulações foram realizadas em três diferentes velocidades de rotação:17,0 rpm (cascata), 31,7 rpm (catarata), 74,6 rpm (centrifugação).

Variáveis de simulação como viscosidade granular, viscosidade granular bulk, pressão de sólidos, função de distribuição radial, entre outras, foram consultadas de modelos presentes na literatura (SYAMLAL E O’BRIEN, 1993. LUN et al., 1984, GIDASPOW 1992).

\section{RESULTADOS E DISCUSSÃO}

$\mathrm{Na}$ abordagem experimental efetuaram-se medidas a quatro diferentes alturas de abandono das esferas, conforme mostrado pela Tabela 1 .

Tabela 1: Coeficiente de restituição das esferas medidos experimentalmente $\left(\mathrm{e}_{\mathrm{ss}}\right)$.

\begin{tabular}{|c|c|c|c|}
\hline Medidas & $\mathrm{H}(\mathrm{cm})$ & $\mathrm{h}(\mathrm{cm})$ & $e_{s s}[-]$ \\
\hline 1 & 30,0 & 8,0 & 0,516 \\
\hline 2 & 29,0 & 7,7 & 0,515 \\
\hline 3 & 25,0 & 8,8 & 0,593 \\
\hline 4 & 24,5 & 7,3 & 0,546 \\
\hline 5 & 21,0 & 7,5 & 0,598 \\
\hline 6 & 20,0 & 8,7 & 0,659 \\
\hline 7 & 14,5 & 5,8 & 0,633 \\
\hline 8 & 15,0 & 5,3 & 0,594 \\
\hline 9 & 15,0 & 6,1 & 0,638 \\
\hline \multicolumn{3}{|c|}{ Média } & $\mathbf{0 , 5 8 8}$ \\
\hline \multicolumn{3}{|c|}{ Desvio Padrão } & 0,052 \\
\hline
\end{tabular}

Nota-se que à medida que se aumenta a altura de abandono da esfera, menor é o valor do coeficiente de restituição. Isto pode ser relacionado à resistência do ar, pois a maiores alturas de abandono, a esfera percorre uma maior trajetória pelo ar até atingir as esferas abaixo, o que faz com que sua velocidade de impacto seja reduzida.

$\mathrm{O}$ valor médio obtido para o coeficiente de restituição foi de $0,588 \pm 0,052$. Este valor está condizente com valores observados em literatura. Rajamani e Venugopal (2001) assumiram como 0,6 o valor do coeficiente de restituição de esferas de aço de diâmetro 50,80 mm e densidade $8000 \mathrm{~kg} / \mathrm{m}^{3}$, enquanto que Hlungwani et al. (2003), adotaram o valor $e_{s s}=$ 0,66 para esferas de aço-cromo de diâmetro $22,24 \mathrm{~mm}$ e densidade $7800 \mathrm{~kg} / \mathrm{m}^{3}$.

A Figura 3 apresenta os resultados obtidos em simulação para a velocidade correspondente ao regime de cascata. Observou-se influências muito discretas no comportamento da carga moedora para os diferentes valores de coeficiente de restituição adotados. 


\section{CXIBEQ 2007 \\ CONGRESSO BRASILEIRO DE ENGENHARIA QUÍMICA EM INICIAÇÃO CIENTÍFICA}

\section{Congresso Brasileiro de Engenharia Química em Iniciação Científica UFSCar - São Carlos - SP 16 a 19 de Julho de 2017}

Figura 3: Frações volumétricas de sólidos observadas numericamente para diferentes valores de coeficiente de restituição durante o regime de cascata $(17,0 \mathrm{rpm})$.

\begin{tabular}{c|c|c|c|}
\hline \multicolumn{5}{c}{ Coeficiente de Restituição } & \multicolumn{1}{c}{$e_{s s}=0,6$} \\
\hline Parede Plano meio & Parede Plano meio & Parede & Plano meio \\
\hline
\end{tabular}

Analogamente, a Figura 4 mostra pouca influência do coeficiente de restituição no perfil de movimentação da carga moedora. Em comparação com a Figura 1, observa-se pouca diferença entre os resultados encontrados e a descrição do regime de cascata presente na literatura.

Figura 4: Frações volumétricas de sólidos observadas numericamente para diferentes valores de coeficiente de restituição durante o regime de catarata $(31,7 \mathrm{rpm})$.

\begin{tabular}{c|c|c|c}
\multicolumn{5}{c}{ Coeficiente de Restituição } \\
\multicolumn{2}{c}{$e_{s s}=0,5$} & $e_{s s}=0,6$ & $e_{s s}=0,7$ \\
\hline Parede Plano meio & Parede Plano meio & Parede Plano meio \\
\hline
\end{tabular}

Notou-se que os valores de coeficiente de restituição são mais influentes no perfil de movimentação da carga moedora quando aplicados a maiores velocidades de operação do moinho. Tal fato pode ser confirmado pela Figura 5, a qual representa os resultados de simulação obtidos para a velocidade de centrifugação (74,6 rpm).

Figura 5: Frações volumétricas de sólidos observadas numericamente para diferentes valores de coeficiente de restituição durante o regime de centrifugação (74,6 rpm).

\begin{tabular}{c|c|c|c}
\hline \multicolumn{3}{c}{ Coeficiente de Restituição } & \multicolumn{2}{c}{$e_{s s}=0,7$} \\
\hline Parede & Plano meio & Parede Plano meio & Parede \\
\end{tabular}




\section{CONCLUSÕES}

A metodologia experimental utilizada mostrou-se adequada para determinação do coeficiente de restituição neste caso. No entanto, ressalta-se a necessidade de investigações futuras em casos mais específicos como, por exemplo, com esferas de diferentes diâmetros ou com a presença de material a ser moído no interstício da colisão.

Dentro do intervalo do coeficiente de restituição estudado, não foi possível observar diferenças significativas nas simulações, especialmente quando são empregadas baixas velocidades de rotação do moinho. Já para altas velocidades de rotação, que neste caso resulta no surgimento do regime de centrifugação, o efeito do coeficiente de restituição se mostrou mais pronunciado devido ao aumento no número de colisões entre as esferas.

\section{REFERENCIAS}

DARTEVELLE, S. Numerical and granulometric approaches to geophysical granular flows. 132 p. Ph. D. thesis, Michigan Technological University, Department of Geological and Mining Engineering, Houghton, Michigan, 2003.

HLUNGWANI, O., RIKHOTSO, J., DONG, H., MOYS, M.H. Futher validation of DEM modeling of milling: effects of liner profile and mill speed. Minerals Engineering, vol. 16, p. 993-998, 2003.

KAPUR, P.C., RANJAN, S., FUERSTENAU, D.W. 1992, A cascade-cataract charge flow model for power draft of tumbling mill. International J. of Mineral Processing., 36, 929.

LUN, C.K.K.; SAVAGE, S.B.; JEFFREY, D.J.; CHEPURNIY N.; Kinetic theories for granular flow: inelastic particles in coquette flow and singly inelastic particles in a general flow field, J. Fluid Mech. v.140, p. 223-256, 1984

MACHADO, M. V. C., 2016, Estudo experimental e numérico da dinâmica de corpos moedores em moinhos de bolas PPGEQ/Faculdade de Engenharia Química da UFU, Uberlândia-MG. (Dissertação de Mestrado), 92 p.

MELLMANN, J, 2001, The transverse motion of solids in rotating cylinders - forms of motion and transition behavior, Powder Technol, 118, 251-270.

NASCIMENTO, S.M., SANTOS, D.A. BARROZO, M.A.S DUARTE, C.R. ,2015, Solids holdup in flighted rotating drums: an experimental and simulation study. Powder Technol, 280, 18-25.

RAJAMANI, R.K., VENUGOPAL, R. 3D simulation of charge motion in tumbling mills by the discrete element method. Powder Technology, vol. 115, p. 157-166, 2001.

SANTOS, D.A., PETRI, I.J., DUARTE, C.R., BARROZO, M.A.S., 2013. Experimental and CFD study of the hydrodynamic behavior in a rotating drum, Powder Technol, 250, 5262. 